\title{
Geometry of Chaos: Advanced computational ap- proach to treating chaotic dynamics of environ- mental radioactivity systems I General Formalism
}

\author{
A.V. Glushkov, V.M. Kuzakon, O.Yu. Khetselius, V.B. Ter- \\ novsky
}

\begin{abstract}
In the paper we go on a development of effective new chaos geomentry and non-linear analysis technique to studying chaotic features of different nature systems. Here there are presented a general for,alism of a chaos-geometrical appraoch to treating, analysing, modelling and forecasting chaotic dynamics of environmental radioactivity systems. A usually, an approach combines together application of the advanced mltifractal formalism, advanced mutual information scheme, Grasberger-Procachi algorythm, Lyapunov exponent's analysis, method of precicted phase trajectories, memory functions method, neural networks algorythms etc.
\end{abstract}

Keywords geometry of chaos, non-linear analysis, radioactivity systems

Mathematics Subject Classification: (2000) 55R01-55B13

\section{UDK 517.9}

\section{Introduction}

In this paper we go on our work on application of the new effective chaos theory and advanced non-linear analysis technique to studying chaotic features of different nature systems. The theoretical basis's of the chaos-geometric combined approach to treating of chaotic behaviour of complex dynamical systems are in details in series of ref. [1-10]. Generally, an approach combines together application of the advanced mutual information scheme, Grassberger-Procachi algory- 
thm, Lyapunov exponent's analysis etc. It is important to note that this approch has been successfully applied to studying dynamics not only mathematical and physical systems. Very impressive application is the investigated dynamics of the atmospheric pollutants concentrations and forecasting their temporal evolution. Besides, in Ref [2] it has been numerically vstudied a chaotic dynamics of the pollutants concentration in some hydroecological, namely, water system. The successful application of new chaos-geometrical approach to studying dynamics of the different nature systems demonstrates its universal character.

Here, starting from our previous works (see, for example [1-7]), we present the possibilities of using a chaos-geometric approach to treating chaotic dynamics of environmental radioactivity systems. In fact, speech is about a perspective application of a chaos geometry tools to treating very important applied problem. Let us remind that hitherto the different mathematical modeling methods deal with serious principal and numerical difficulties under studying the key radioecological transfer and effects such as radionuclide cycles in the ecosystems, radionuclide transfer to biota in inland waters, biological effects of radiation exposure to living organisms etc.

\section{Chaos-geometric approach to treating chaotic dynamics with application to environmental radioactivity systems}

As our approach has been presented earlier [1-11], here we are limited only by the key moments. In fact the main task is resulted in reformulation and introducing the key parameters in all computational methods, algorythms and schemes of a chaos-geometrical appraoch. Naturally, the numerical and computational features of the methods remain unchanged. So here we only deal with formal task of redefinition and reformulation of the key dynamical parameters.

Accordingly to $[1,8,11]$ let us formally consider scalar measurements for any environmental radioactivity systems $s(n)=s\left(t_{0}+n \Delta t\right)=s(n)$, where $t_{0}$ is the start time, $\Delta t$ is the time step, and is $n$ the number of the measurements. Further it is necessary to reconstruct phase space using as well as possible information contained in the $s(n)$. Such a reconstruction results in a certain set of $d$-dimensional vectors $\mathbf{y}(n)$ replacing the scalar measurements. Packard et al. [14] introduced the method of using time-delay coordinates to reconstruct the phase space of an observed dynamical system. The direct use of the lagged variables $s(n+\tau)$, where $\tau$ is some integer to be determined, results in a coordinate system in which the structure of orbits in phase space can be captured. 


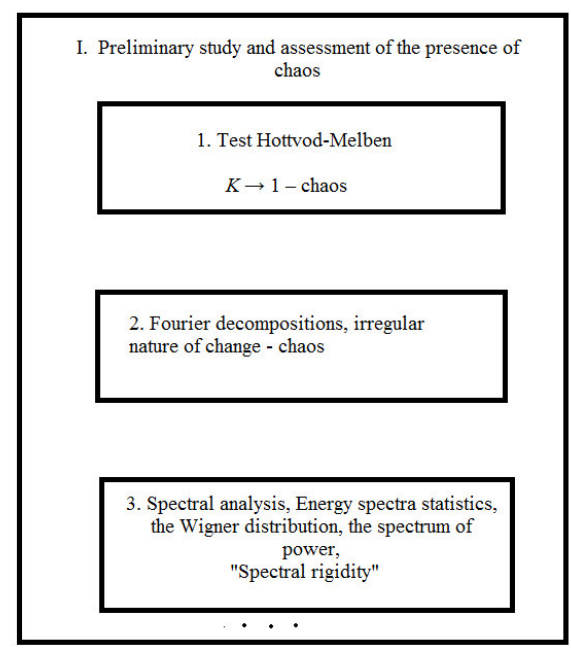

$\Downarrow$

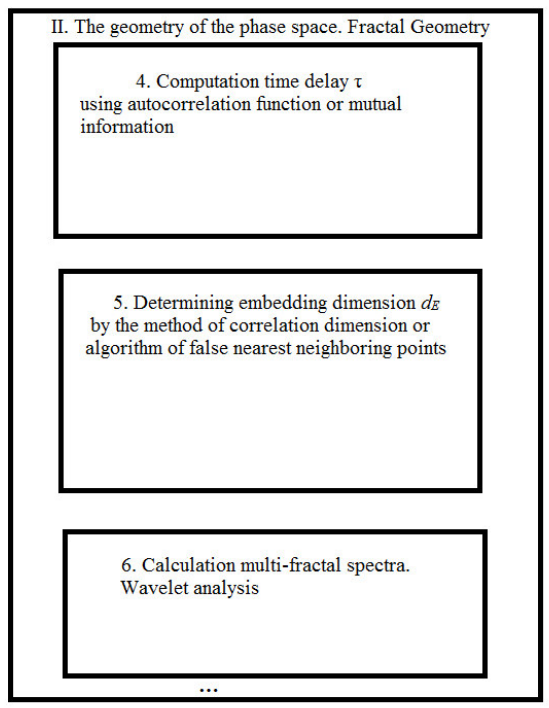




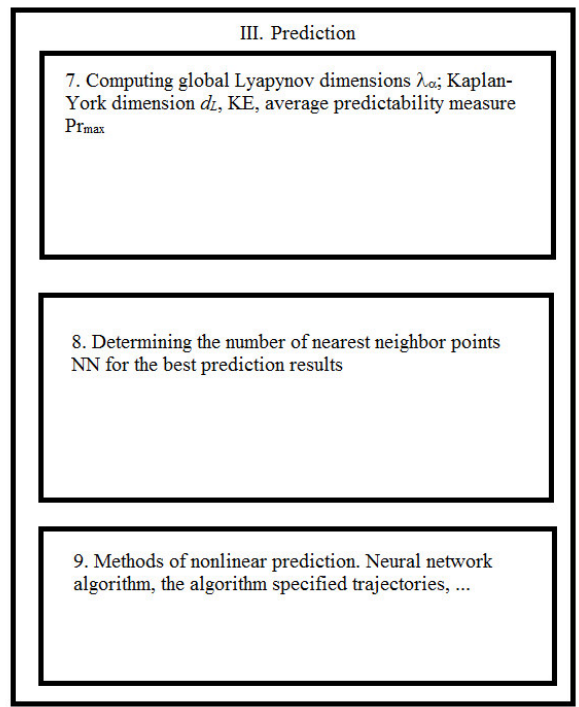

Figure 1. Chaos and neural network-geometric approach to nonlinear analysis and forecast chaotic dynamics processes in environmental radioactivity systems.

Then using a collection of time lags to create a vector in $d$ dimensions,

$$
\mathbf{y}(n)=[s(n), s(n+\tau), s(n+2 \tau), \ldots, s(n+(d-1) \tau)]
$$

the required coordinates are provided. In a nonlinear system, the $s(n+j \tau)$ are some unknown nonlinear combination of the actual physical variables that comprise the source of the measurements. The dimension $d$ is called the embedding dimension, $d_{E}$.

According to Mane and Takens [12], any time lag will be acceptable is not terribly useful for extracting physics from data. If $\tau$ is chosen too small, then the coordinates $s(n+j \tau)$ and $s(n+(j+1) \tau)$ are so close to each other in numerical value that they cannot be distinguished from each other. Similarly, if $\tau$ is too large, then $s(n+j \tau)$ and $s(n+(j+1) \tau)$ are completely independent of each other in a statistical sense. Also, if $\tau$ is too small or too large, then the correlation dimension of attractor can be under- or overestimated respectively [3]. It is therefore necessary to choose some intermediate (and more appropriate) position between above cases. First approach is to compute the linear autocorrelation function

$$
C_{L}(\delta)=\frac{\frac{1}{N} \sum_{m=1}^{N}[s(m+\delta)-\bar{s}][s(m)-\bar{s}]}{\frac{1}{N} \sum_{m=1}^{N}[s(m)-\bar{s}]^{2}},
$$

where $\bar{s}=\frac{1}{N} \sum_{m=1}^{N} s(m)$ 
and to look for that time lag where $C_{L}(\delta)$ first passes through zero. This gives a good hint of choice for $\tau$ at that $s(n+j \tau)$ and $s(n+(j+1) \tau)$ are linearly independent. However, a linear independence of two variables does not mean that these variables are nonlinearly independent since a nonlinear relationship can differs from linear one. It is therefore preferably to utilize approach with a nonlinear concept of independence, e.g. the average mutual information. Briefly, the concept of mutual information can be described as follows. Let there are two systems, $A$ and $B$, with measurements $a_{i}$ and $b_{k}$. The amount one learns in bits about a measurement of $a_{i}$ from measurement of $b_{k}$ is given by arguments of information theory $[3,7]$

$$
I_{A B}\left(a_{i}, b_{k}\right)=\log _{2}\left(\frac{P_{A B}\left(a_{i}, b_{k}\right)}{P_{A}\left(a_{i}\right) P_{B}\left(b_{k}\right)}\right),
$$

where the probability of observing $a$ out of the set of all $A$ is $P_{A}\left(a_{i}\right)$, and the probability of finding $b$ in a measurement $B$ is $P_{B}\left(b_{i}\right)$, and the joint probability of the measurement of $a$ and $b$ is $P_{A B}\left(a_{i}, b_{k}\right)$. The mutual information $I$ of two measurements $a_{i}$ and $b_{k}$ is symmetric and non-negative, and equals to zero if only the systems are independent. The average mutual information between any value $a_{i}$ from system $A$ and $b_{k}$ from $B$ is the average over all possible measurements of $I_{A B}\left(a_{i}, b_{k}\right)$,

$$
I_{A B}(\tau)=\sum_{a_{i}, b_{k}} P_{A B}\left(a_{i}, b_{k}\right) I_{A B}\left(a_{i}, b_{k}\right)
$$

To place this definition to a context of observations from a certain physical system, let us think of the sets of measurements $s(n)$ as the $A$ and of the measurements a time lag $\tau$ later, $s(n+\tau)$, as $B$ set. The average mutual information between observations at $n$ and $n+\tau$ is then

$$
I_{A B}(\tau)=\sum_{a_{i}, b_{k}} P_{A B}\left(a_{i}, b_{k}\right) I_{A B}\left(a_{i}, b_{k}\right)
$$

Now we have to decide what property of $I(\tau)$ we should select, in order to establish which among the various values of $\tau$ we should use in making the data vectors $\mathbf{y}(n)$. One could remind that the autocorrelation function and average mutual information can be considered as analogues of the linear redundancy and general redundancy, respectively, which was applied in the test for nonlinearity. The general redundancies detect all dependences in the time series, while the linear redundancies are sensitive only to linear structures. Further, a possible nonlinear nature of process resulting in the vibrations amplitude level variations can be concluded. 
The goal of the embedding dimension determination is to reconstruct a Euclidean space $R^{d}$ large enough so that the set of points $d_{A}$ can be unfolded without ambiguity. In accordance with the embedding theorem, the embedding dimension, $d_{E}$, must be greater, or at least equal, than a dimension of attractor, $d A$, i.e. $d_{E}>d_{A}$. However, two problems arise with working in dimensions larger than really required by the data and time-delay embedding $[1,7,13,19]$. First, many of computations for extracting interesting properties from the data require searches and other operations in $R^{d}$ whose computational cost rises exponentially with $d$. Second, but more significant from the physical point of view, in the presence of noise or other high dimensional contamination of the observations, the extra dimensions are not populated by dynamics, already captured by a smaller dimension, but entirely by the contaminating signal. In too large an embedding space one is unnecessarily spending time working around aspects of a bad representation of the observations which are solely filled with noise. It is therefore necessary to determine the dimension $d_{A}$. There are several standard approaches to reconstruct the attractor dimension (see, e.g., [3,7-12]), but let us consider in this study two methods only. The correlation integral analysis is one of the widely used techniques to investigate the signatures of chaos in a time series. The analysis uses the correlation integral, $C(r)$, to distinguish between chaotic and stochastic systems. To compute the correlation integral, the algorithm of Grassberger and Procaccia [17] is the most commonly used approach. According to this algorithm, the correlation integral is

$$
\begin{gathered}
C(r)=\lim _{N \rightarrow \infty} \frac{2}{N(n-1)} \sum_{\substack{i, j \\
(1 \leq i<j \leq N)}} H\left(r-\left\|y_{i}-y_{j}\right\|\right) \\
\end{gathered}
$$

where $H$ is the Heaviside step function with $H(u)=1$ for $u>0$ and $H(u)=0$ for $u \leq, r$ is the radius of sphere centered on $\mathbf{y}_{i}$ or $\mathbf{y}_{j}$, and $N$ is the number of data measurements. If the time series is characterized by an attractor, then the integral $C(r)$ is related to the radius $r$ given by

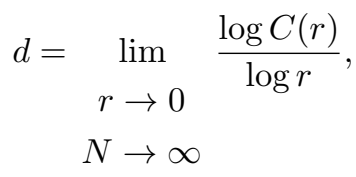

where $d$ is correlation exponent. The method of surrogate data is one othe important methods of a general chaos-geometric approach [1-22]. It is an approach that makes use of the substitute data generated in accordance to the 
probabilistic structure underlying the original data [3,7-11]. This means that the surrogate data possess some of the properties, such as the mean, the standard deviation, the cumulative distribution function, the power spectrum, etc., but are otherwise postulated as random, generated according to a specific null hypothesis. Here, the null hypothesis consists of a candidate linear process, and the goal is to reject the hypothesis that the original data have come from a linear stochastic process. One reasonable statistics is obtained as follows. If we denote $Q_{\text {orig }}$ as the statistic computed for the original time series and $Q_{s i}$ for $i$ th surrogate series generated under the null hypothesis and let $\mu_{s}$ and $\sigma_{s}$ denote, respectively, the mean and standard deviation of the distribution of $Q_{s}$, then the measure of significance $S$ is given by $S=\frac{\left|Q_{\text {orig }}-\mu_{s}\right|}{\sigma_{s}}$. An $S$ value of $\sim 2$ cannot be considered very significant, whereas an $S$ value of $\sim 10$ is highly significant. To detect nonlinearity in the amplitude level data, the one hundred realizations of surrogate data sets were generated according to a null hypothesis in accordance to the probabilistic structure underlying the original data. Often, a significant difference in the estimates of the correlation exponents, between the original and surrogate data sets, can be observed. In the case of the original data, a saturation of the correlation exponent is observed after a certain embedding dimension value (i.e., 6), whereas the correlation exponents computed for the surrogate data sets continue increasing with the increasing embedding dimension. The high significance values of the statistic indicate that the null hypothesis (the data arise from a linear stochastic process) can be rejected and hence the original data might have come from a nonlinear process. It is worth consider another method for determining $d_{E}$ that comes from asking the basic question addressed in the embedding theorem: when has one eliminated false crossing of the orbit with itself which arose by virtue of having projected the attractor into a too low dimensional space? By examining this question in dimension one, then dimension two, etc. until there are no incorrect or false neighbours remaining, one should be able to establish, from geometrical consideration alone, a value for the necessary embedding dimension. Advanced version is presented in Ref. [3]

The LE are the dynamical invariants of the nonlinear system. In a general case, the orbits of chaotic attractors are unpredictable, but there is the limited predictability of chaotic physical system, which is defined by the global and local LE. A negative exponent indicates a local average rate of contraction while a positive value indicates a local average rate of expansion. In the chaos theory, the spectrum of LE is considered a measure of the effect of perturbing the 
initial conditions of a dynamical system. In fact, if one manages to derive the whole spectrum of the LE, other invariants of the system, i.e. KE and attractor's dimension can be found. The KE, $K$, measures the average rate at which information about the state is lost with time. An estimate of this measure is the sum of the positive LE. The inverse of the KE is equal to an average predictability. Estimate of dimension of the attractor is provided by the Kaplan and Yorke conjecture:

$$
d_{L}=j+\frac{\sum_{\alpha=1}^{j} \lambda_{\alpha}}{\left|\lambda_{j+1}\right|},
$$

where $j$ is such that $\sum_{\alpha=1}^{j} \lambda_{\alpha}>0$ and $\sum_{\alpha=1}^{j+1} \lambda_{\alpha}<0$, and the LE $\lambda_{\alpha}$ are taken in descending order. There are a few approaches to computing the LE. One of them computes the whole spectrum and is based on the Jacobi matrix of system [3]. In the case where only observations are given and the system function is unknown, the matrix has to be estimated from the data. To calculate the spectrum of the LE from the amplitude level data, one could determine the time delay $\tau$ and embed the data in the four-dimensional space. In this point it is very important to determine the Kaplan-Yorke dimension and compare it with the correlation dimension, defined by the Grassberger-Procaccia algorithm. The estimations of the KE and average predictability can further show a limit, up to which the amplitude level data can be on average predicted.

\section{Conclusions}

We briefly considered the theoretical basis of our new chaos geometry and nonlinear analysis technique to studying chaotic features of different nature systems, in particular, with application to treating, analysing, modelling and forecasting chaotic dynamics of environmental radioactivity systems. A usually, an approach combines together application of the advanced mltifractal formalism, advanced mutual information scheme, Grrasberger-Procachi algorythm, Lyapunov exponent's analysis, method of precicted phase trajectories, memory functions method, neural networks algorythms etc. In the next paper we will present the results of the numerical analysis for array of values of radon flux from the Earth's surface, calculated from the experimental data of time series of radon fields and thus firstly demonstrate a perspectives of using new chaos geomentry and nonlinear analysis technique in studying dynamical and topological characteristics of chaotic dynamics in the environmental radioactivity systems. 


\section{References}

1. Glushkov A.V., Ternovsky V.B., Buyadzhi V.V., Prepelitsa G.P., A Geometry of a Relativistic Quantum Chaos: New approach to dynamics of quantum systems in electromagnetic field and uniformity and charm of a chaos// Proc// Int. Geom. Centre.- 2014.Vol.7,N4.-P.60-71.

2. Glushkov A.V., Buyadzhi V.V., Ponomarenko E.L., Geometry of Chaos: Advanced approach to treating chaotic dynamics in some nature systems// Proc. Int. Geom. Centre.2014.-Vol.7,N1.-P.24-29

3. Glushkov A.V., Kuzakon' V.M., Khetselius O.Yu., Prepelitsa G.P. and Svinarenko A.A., Geometry of Chaos: Theoretical basis's of a consistent combined approach to treating chaotic dynamical systems and their parameters determination// Proc. Int. Geom. Centre.-2013.-Vol.6,N1.-P.6-12.

4. Glushkov A.V., Khetselius O.Yu., Svinarenko A.A., Prepelitsa G.P.Energy approach to atoms in a laser field and quantum dynamics with laser pulses of different shape///In: Coherence and Ultrashort Pulsed Emission, Ed. Duarte F. J. (Intech, Vienna).-2011.P.159-186.

5. Bunyakova Yu.Ya., Glushkov A.V.,Fedchuk A.P., Serbov N.G., Svinarenko A.A., Tsenenko I.A., Sensing non-linear chaotic features in dynamics of system of couled autogenerators: standard multifractal analysis// Sensor Electr. and Microsyst. Techn.-2007.-N1.-P.14-17.

6. Glushkov A.V., Kuzakon V.M., Buyadzhi V.V., Solyanikova E.P., Geometry of Chaos: Advanced computational approach to treating chaotic dynamics of some hydroecological systems// Proc. Int. Geom. Centre.-2015.-Vol.8,N1.-P.93-99.

7. Glushkov A.V., Kuzakon V.M., Bunyakova Yu.Ya., Buyadzhi V.V., Geometry of Chaos: Advanced computational approach to treating chaotic dynamics of some hydroecological systems II// // Proc. Int. Geom. Centre.-2015.-Vol.8,N2.-P.91-96.

8. Glushkov A.V., Prepelitsa G.P., Lepikh Ya.I., Buyadzhi V.V., Ternovsky V.B., Zaichko P.A., Chaotic dynamics of non-linear processes in atomic and molecular systems in electromagnetic field and semiconductor and fiber laser devices: new approaches, uniformity and charm of chaos// Sensor Electronics and Microsystems Techn.-2014.-Vol.11,N4.-P.43-57.

9. Glushkov A.V., Khetselius O.Yu., Bunyakova Yu.Ya., Prepelitsa G.P., Solyanikova E.P., Serga E.N., Non-linear prediction method in short-range forecast of atmospheric pollutants: low-dimensional chaos// Dynamical Systems - Theory and Applications. - Lodz: Lodz Univ. Press (Poland). -2011.- LIF111 (6p.).

10. Glushkov A.V., Bunyakova Yu.Ya., Zaichko P.A., Geometry of Chaos: Consistent combined approach to treating chaotic dynamics atmospheric pollutants and its forecasting// Proc. of Int. Geometry Center.-2013.-Vol.6,N3.-P.6-14.

11. Glushkov A.V., Svinarenko A.A., Buyadzhi V.V., Zaichko P.A., Ternovsky V.B., Chaosgeometric attractor and quantum neural networks approach to simulation chaotic evolutionary dynamics during perception process// Advances in Neural Networks, Fuzzy Systems and Artificial Intelligence, Series: Recent Advances in Computer Engineering (Gdansk,EU).-2014.-Vol.21.-P.143-150.

12. Parovik P.I., Shevtsov B.M.: Modeling a radon transfer process in mediums with a fracral structure // Mathematical Modelling.-2009.-Vol.21,N8.-P.30-36.

13. KoP.ak K., Saylan L., Sen O., Nonlinear time series prediction of $\mathrm{O}_{3}$ concentration in CityplaceIstanbul.. AtmosphericEnvironment (Elsevier) 34, 2000, 1267-1271.

14. Packard N., Crutchfield J., Farmer J., Shaw R., Geometry from time series// Phys.Rev.Lett-1988.-Vol.45.-P.712-716.

15. Gallager R.G., Information theory and reliable communication, Wiley, New York.-1986.

16. Kennel M., Brown R., Abarbanel H., Determining embedding dimension for phase-space reconstruction using geometrical construction//Phys.Rev.A.-1992.-Vol.45.-P.3403-3411.

17. Grassberger P., Procaccia I., Measuring the strangeness of strange attractors// Physica D.-1983.-Vol.9.-P.189-208.

18. Fraser A., Swinney H., Independent coordinates for strange attractors from mutual information// Phys Rev A.-1986.-Vol.33.-P.1134-1140.

19. Takens F (1981) Detecting strange attractors in turbulence. In: Rand DA, Young LS (eds) Dynamical systems and turbulence, Warwick 1980. (Lecture notes in mathematics No 898). Springer, Berlin Heidelberg New York, pp 366-381

20. Mane R (1981) On the dimensions of the compact invariant sets of certain non-linear maps. In: Rand DA, Young LS (eds) Dynamical systems and turbulence, Warwick 1980. (Lecture notes in mathematics No 898). Springer, Berlin Heidelberg N.-Y., p. 230-242 
21. Sano M, Sawada Y (1985) Measurement of the Lyapunov spectrum from a chaotic time series / /Phys Rev.Lett.-1995.-Vol.55.-P.1082-1085

22. Theiler J., Eubank S., Longtin A., Galdrikian B., Farmer J., Testing for nonlinearity in time series: The method of surrogate data// Physica D.-1992.-Vol.58.-P.77-94.

23. Kaplan J.L., Yorke J.A., Chaotic behavior of multidimensional difference equations, in: Peitgen H.-O., Walter H.-O. (Eds.), Functional Differential Equations and Approximations of Fixed Points. Lecture Notes in Mathematics No. 730. Springer, Berlin.-1979.-pp.204-227.

\author{
A.V. Glushkov, V.M. Kuzakon, O.Yu. Khetselius, V.B. Ternovsky \\ Mathematics Department \\ Odessa State Environmental University, Ukraine \\ E-mail: (fdirac13@mail.ru )
}

